\title{
The effect of self-selecting the number of repetitions on motor performance and psychological outcomes
}

\author{
Aviv Emanuel ${ }^{1,2,3} \cdot$ Itai Har-Nir ${ }^{2,3} \cdot$ Isaac Isur Rozen Smukas ${ }^{2,3} \cdot$ Israel Halperin ${ }^{2,3}$
}

Received: 2 May 2020 / Accepted: 4 August 2020 / Published online: 10 August 2020

(c) Springer-Verlag GmbH Germany, part of Springer Nature 2020

\begin{abstract}
In resistance-training, the number of repetitions can be either fixed and predetermined (e.g., 3 sets of 10 repetitions), or selected by the trainee during ongoing sets (e.g., 3 sets of 8-12 repetitions). The first approach is more goal-focused while the latter is more autonomy-focused. Here we compared these two approaches in motor performance and psychological outcomes. Nineteen resistance-trained participants (10-males) first completed one repetition-maximum (RM) tests in the barbell-squat and bench-press, and were familiarized with the isometric mid-thigh pull (IMTP). In the next two counterbalanced sessions, participants completed two sets of the squat and bench-press using 70\%1RM, and two sets of the IMTP. In the predetermined session, participants completed 10 repetitions in all sets, and in the self-selected session, participants chose how many repetitions to complete out of an 8-12 range. Bar-velocity was measured in the squat and bench-press, and force production in the IMTP. Enjoyment, perceived-autonomy, and approach-preferences were collected post-sessions. We observed comparable bar-velocity, force production, and enjoyment in both conditions (all $\mathrm{BF}_{01}>2.1$ ), and an even approachpreferences split. However, in the self-selected condition, participants demonstrated considerable variability in the number of repetitions and reported greater perceived-autonomy. Given the similarities between approaches, both can be used with this cohort based on their personal-preference.
\end{abstract}

\section{Introduction}

Prescribing the number of repetitions to complete per set for an exercise, is a key variable in designing resistance-training (RT) programs. Traditionally, the number of prescribed repetitions is predetermined and fixed. That is, the number of repetitions is decided upon before the session or set-initiation (e.g., 3 sets of 10 repetitions, or 5 sets of 5 repetitions) (DeLorme 1945; Sands, Wurth, \& Hewit 2012; Todd, Shurley, \& Todd 2012). Following this approach has a prominent

Electronic supplementary material The online version of this article (https://doi.org/10.1007/s00426-020-01402-4) contains supplementary material, which is available to authorized users.

Israel Halperin

ihalperin@tauex.tau.ac.il

1 School of Psychological Sciences, Tel-Aviv University, Tel-Aviv, Israel

2 School of Public Health, Sackler Faculty of Medicine, Tel-Aviv University, Tel-Aviv, Israel

3 Sylvan Adams Sports Institute, Tel Aviv University, Tel-Aviv, Israel psychological benefit-it provides one with a clear goal. According to the goal-specificity theory (Locke \& Latham 1990) a concrete, attainable goal for any task increases people's motivation in its performance, compared to tasks of identical timespan in which no concrete goal is specified (Smith, Locke, \& Barry 1990). Accordingly, when people know exactly what to expect, they tend to apply greater efforts (Billaut, Bishop, Schaerz \& Noakes 2011; Halperin, Aboodarda, Basset, Byrne, \& Behm 2014a, b; Hanson \& Buckworth 2015; Wingfield, Marino, \& Skein 2018). For example, people run (Hanson \& Buckworth 2015) and cycle (Wingfield, Marino, \& Skein 2018) faster when they know how long they need to run or cycle for, and apply greater forces when they know how many repetitions they need to complete, compared to unknown task endpoints (Halperin, Aboodarda, Basset, Byrne, \& Behm 2014a, b).

The number of repetitions can also be prescribed out of a range (e.g., 3 sets of 8 to 12 repetitions, or 5 sets of 4-6 repetitions) (American College of Sports Medicine 2013). Although the range approach is advocated in resistancetraining guidelines (Fragala et al. 2019; American College of Sports Medicine 2013; Nelson et al. 2007; Sands, Wurth, \& Hewit 2012), it is not clear how it should be implemented. 
On the one hand, the number of repetitions can be determined prior to set- or session-initiation. For example, prescribing 12 repetitions in the first set and 8 in the third set (American College of Sports Medicine 2013). This implementation strategy is in many ways similar to the fixed and predetermined approach, as it does not allow practitioners to evaluate their abilities during ongoing sets, and choose the number of repetitions accordingly. On the other hand, the range approach can be implemented by allowing trainees to choose how many repetitions to complete during the set itself, based on various psychophysiological inputs (e.g., motivation, perception of effort, etc.). Here we refer to the latter prescription strategy as the self-selected approach.

The self-selected approach has several benefits. First, it better accounts for between and within-participant variability in performance. For example, prescribing one with 10 repetitions using $70 \%$ of one repetition maximum (RM) in a given exercise can be easier to complete by one person and harder for another (Richens \& Cleather 2014; Shimano et al. 2006). Similarly, for the same person, performing 10 repetitions can be easier to complete on some days, and harder on others. By allowing one to choose the number of repetitions within a range, trainees can regulate their effort, which better accounts for between- and within-participant variability in performance. Second, choice provision within a training session is known to improve motor learning and performance among a range of populations and outcomes (Halperin, Wulf, Vigotsky, Schoenfeld, \& Behm 2018; Sanli, Patterson, Bray, \& Lee 2013; Teixeira, Carraça, Markland, Silva, \& Ryan 2012; Wulf \& Lewthwaite 2016). For example, Halperin, Chapman, Martin, Lewthwaite, and Wulf (2017) found that competitive boxers punched harder and faster when given a choice regarding the order of delivered punches in a combination, compared to a predetermined punching order. Finally, choice provision leads people to report greater levels of enjoyment following a training session, and increases the likelihood of adhering to the exercise program, compared to a no-choice group (Fortier, Sweet, O'Sullivan, \& Williams 2007; Silva et al. 2011). The capacity to decide for oneself increases the sense of autonomy, which facilitates a higher level of intrinsic motivation for action. Indeed, the capacity to decide for oneself is a key component in prominent theories of motivation including the self-determination theory (Deci \& Ryan 2000, 2002) and the optimizing performance through intrinsic motivation and attention for learning theory (Wulf \& Lewthwaite 2016).

To date, no study has compared the effects of a fixed and self-selected approaches on performance and self-report measures in a resistance-training setting. Such an investigation can hold high theoretical and practical value. Accordingly, in this study, resistance-trained participants performed two resistance-training sessions composed of two sets of the bench-press and the squat using $70 \%$ of their 1RM, followed by two sets with the isometric mid-thigh pull (IMTP). On one day participants completed 10 repetitions across exercises and sets (fixed condition), and on the other day they chose how many repetitions to complete out of an 8-12 range during ongoing sets (self-selected condition). We compared the effects of both conditions on (1) performance measures, including bar-velocity and force output and; (2) psychological measures, including perceived autonomy, enjoyment levels, and preference of one approach over the other. Of note, we considered preference for one approach over the other as a motivational measure, similar to those used previously in the self-determination theory literature (Ryan, Rigby, \& Przybylski 2006; Standage, Duda, \& Ntoumanis 2005).

\section{Materials and methods}

\section{Participants}

The planned sample-size of 25 resistance trained participants was determined as a compromise between our laboratory's resources, and our expectancy of the required sample size to allow $80 \%$ power for medium sized effects in paired sample $\mathrm{t}$-test for performance measures and enjoyment. However, we were forced to terminate data collection prematurely due to the Coronavirus outbreak. The final sample consisted of 19 participants who completed both experimental sessions (Table 1). Inclusion criteria included healthy participants between the ages of 18 and 45 with at least six months of resistance-training experience. Participants also had to be accustomed to performing the barbell-squat and bench-press using a variety of repetition ranges (e.g., 8-15 repetitions). Each participant signed an informed consent on the first day. This study was approved by the institutional review board.

\section{Measures}

\section{Performance measures}

Average concentric velocity $\left(\mathrm{m} \mathrm{s}^{-1}\right)$ of the barbell was measured using GymAware PowerTool (GymAware, Canberra, Australia) linear position transducer. The GymAware was synced with a tablet application that displayed the average concentric velocity of each repetition and exported the data as an Excel file. The device was used according to the instructions of the manufacturers so that when it was attached to the barbell a perpendicular angle was achieved during all lifts.

Peak force $(\mathrm{N})$ production in the IMTP was collected by AMTI AccuPower portable force plate (AMTI, Massachusetts, USA). The force plate synchronized with a Dell laptop and analyzed with a LabView (v2017, National Instruments, 
Table 1 General demographics

\begin{tabular}{lll}
\hline & $\begin{array}{l}\text { Females }(n=9) \\
\text { Mean } \pm \text { SD (range) }\end{array}$ & $\begin{array}{l}\text { Males }(n=10) \\
\text { Mean } \pm \text { SD (range) }\end{array}$ \\
\hline Age & $29.2 \pm 3.4(24-34)$ & $31.6 \pm 5.8(23-44)$ \\
Height $(\mathrm{cm})$ & $168 \pm 7.5(153-170)$ & $178.5 \pm 5.3(171-186)$ \\
Weight $(\mathrm{kg})$ & $65.4 \pm 11.0(49-82)$ & $78.7 \pm 6.2(70.9-89)$ \\
Experience in RT (years) & $4.4 \pm 2.4(1-4)$ & $8.6 \pm 6.2(3-19)$ \\
Mean workouts per week & $3.4 \pm 1.1(2-5)$ & $3.3 \pm 1.0(2-5)$ \\
1RM barbell bench press $(\mathrm{kg})$ & $45.5 \pm 10.3(30-65)$ & $92.7 \pm 10.4(77.5-110)$ \\
1RM/ Bodyweight bench press & $0.70 \pm 0.16(0.36-0.91)$ & $1.37 \pm 0.26(0.95-1.16)$ \\
Average velocity 1RM barbell bench press $\left(\mathrm{m} \mathrm{s}^{-1}\right)$ & $0.16 \pm 0.09(0.07-0.32)$ & $0.16 \pm 0.02(0.12-0.20)$ \\
1RM barbell squat $(\mathrm{kg})$ & $71.6 \pm 18.0(50-110)$ & $108.5 \pm 24.1(75-160)$ \\
1RM/bodyweight squat & $1.1 \pm 0.23(0.80-1.4)$ & $1.3 \pm 0.27(0.96-1.86)$ \\
Average velocity 1RM barbell squat $\left(\mathrm{m} \mathrm{s}^{-1}\right)$ & $0.21 \pm 0.06(0.09-0.31)$ & $0.27 \pm 0.04(0.19-0.34)$ \\
\hline
\end{tabular}

TX, USA). Force data were sampled at $500 \mathrm{~Hz}$ via an analog to digital converter (16-Bit, $250 \mathrm{kS} / \mathrm{s}$ National Instruments, Austin, TX, USA). The repetitions were automatically recognized by a code, which set to include force data higher than 1.5 of one's body weight, detected as the peak force measured when the participant first stood on the force plate. Next, the code omitted $500 \mathrm{~ms}$ from the beginning and ending of each repetition to reduce noise.

\section{Self-report measures}

Perception of autonomy was measured using a modified version of the basic psychological needs in exercise questionnaire (Vlachopoulos, Ntoumanis, \& Smith 2010). Participants rated three items on a Likert scale which ranged from 1 ("I don't agree at all") to 5 ("I totally agree"). The items were presented and explained to the participants using an A4 sheet: "1. The way I exercised today is aligned with my choices and preference", " 2 . I feel the way I exercised today is the way I want to exercise", and "3. I feel like I could make decisions regarding how I exercised today". We note that we expected that participants would provide higher ratings in this questionnaire under the self-selected condition, but were unsure about the extent of differences between the two conditions.

In order to rate session enjoyment, participants were presented the following question after the fixed session: "How much did you enjoy today's session? In your answer, please focus on the fact that the number of repetitions you performed was fixed and predetermined to be 10 " and the following question after the range sessions: "How much did you enjoy today's session? In your answer, please focus on the fact that the number of repetitions you performed was selected out of an 8-12 range". Participants indicated their answer using a visual analog scale (VAS), ranging from "0" ("not at all") to "100" ("extremely enjoyable"), which included vertical lines on each 10 units (see Online Resource). We chose to utilize this scale because (1) it can be easily implemented and acted upon in practice by coaches. For example, based on the trainee's answers, coaches may decide to modify certain aspects of the training sessions; (2) we preferred to use VAS in contrast to Likert based exercise enjoyment scales (e.g., Stanley, Williams, \& Cumming 2009), because VAS scales are better suited to be treated as a continuous variable in statistical analyses.

In order to rate participants' preference between the two approaches, participants were presented with the following question immediately after the last session and $24 \mathrm{~h}$ afterwards: "Out of the two training sessions you performed, which approach do you prefer performing and to what extent?". Participants indicated their answer using a VAS scale, ranging from "-100" ("absolute preference for the fixed approach") to "100" ("absolute preference for the range approach"), that includes vertical lines on each 20 units (see Online Resource).

\section{Procedures}

The study consisted of three testing sessions with a minimum of three and a maximum of ten days between sessions. The first session consisted of 1RM tests for the barbell-backsquat followed by the barbell-bench-press exercise, and a familiarization with the IMTP exercise, questionnaires, and experimental procedure. In the next two sessions, participants performed two sets of each exercise: squat, benchpress (using $70 \%$ of $1 \mathrm{RM}$ ), and IMTP. On the fixed session, participants performed 10 repetitions in all exercises and sets. On the range session, participants chose to perform any number of repetitions between 8 and 12 in all exercises and sets. We employed this range as it can be found in numerous guidelines (Nelson et al. 2007; Sands, Wurth, \& Hewit 2012). We asked participants to decide how many 
repetitions to complete within this range during the set rather than before initiating it. We explained that when making this decision, participants should account to how they feel, as they would do in their regular day-to-day training. On days that they feel refreshed and motivated, they may push themselves harder, and in this study, they may wish to complete more repetitions out of the given range (e.g., complete 11 or 12 repetitions). Conversely, on days that they feel fatigued and demotivated, they may hold themselves back, and in this study, they may wish to complete fewer repetitions out of the given range (e.g., complete 8 or 9 repetitions). At the end of each session, participants rated their perception of autonomy and enjoyment levels. Following the last session and $24 \mathrm{~h}$ afterwards, participants reported which of the two training routines they prefer following.

\section{RM tests and familiarization (session 1)}

In the beginning of the session, participants were weighed, indicated their height, age and experience in strength training, and were briefed on the study's aims. All participants then performed a squat to an approximate fixed knee angle using a box, which height was adjusted individually in the beginning of the session (mean knee angle $=118.31$, $\mathrm{SD}=4.76$ ). During the bench press, participants' preferred grip and body position were recorded and maintained throughout the study. In each repetition the bar must have lightly touched participants' chest prior to the concentric portion of the lift to be counted. Participants then performed a structured warm-up protocol consisting of dynamic stretching and callisthenic exercises, and a 5-min individualized warm-up. This warm-up protocol was identical in all sessions. Upon completion of the warm-up, participants performed the barbell-squat 1RM protocol followed by the barbell-bench 1RM protocol. Briefly, the 1RM protocol consisted of a gradual load-progression towards an estimated $1 \mathrm{RM}$ weight indicated by the participants. The increase in weight towards the actual 1RM was decided by the participants and investigators with 3-5 min of rest between 1RM attempts. After performing the 1RM squat and bench-press, IMTP measurements took place. Bar height was adjusted and secured using ratchet straps, to achieve a knee and hip angle of $125^{\circ}-145^{\circ}$ and $140^{\circ}-150^{\circ}$, respectively, as participants stood on the middle of the force plate (Comfort, Dos'Santos, Beckham, Stone, Guppy, \& Haff 2019). Given the repetitive nature of this task, all participants were requested to use the same hand wraps to reduce muscular fatigue of the wrist flexors.

\section{Experimental sessions (sessions 2-3)}

In the beginning of each session, participants were reminded of the four self-report measures and performed the general warm-up protocol. Participants then performed a specific warm-up procedure prior to performing two sets in the squat, two sets in the bench press and two sets of the IMTP. In the squat and bench-press exercises, participants lifted $70 \%$ of $1 \mathrm{RM}$ and were asked to complete the concentric phase of each repetition as fast as possible as bar-velocity was measured. In the IMTP participants were asked to apply as much force as they possibly can in each repetition against the force plate. Two minutes of rest were provided between sets and exercises. Bar-velocity was recorded during the performance of the squat and bench-press exercises, and force production was recorded during the performance of the IMTP. All sessions were performed in the same facilities, ran by the same two experimenters at approximately the same hour of the day $( \pm 2 \mathrm{~h})$. No verbal encouragement was provided throughout the sessions. Participants were asked to refrain from an intense training session $24 \mathrm{~h}$ prior to testing days that may lead to performance decrements and muscle soreness. Participants were also asked to avoid a heavy meal and caffeinated drinks or supplements at least $3 \mathrm{~h}$ before testing sessions. The two experimental sessions were counterbalanced and randomized between participants.

\section{Statistical analysis}

\section{Performance measures}

We compared the bar-velocity and force outcomes between conditions using two approaches. First, we analyzed the extent by which bar-velocity or force decreased during each set, and whether this trend differs between conditions, in which all repetitions were included in the analysis, despite not necessarily being the same in both conditions. We tested linear, and quadratic mixed models for each exercise with bar-velocity as the dependent variable and repetition number as the predictor, nested within participants. We examined the best-fitted trend by comparing the linear and quadratic models for the best goodness-of-fit, as indicated by the deviance statistic. We then examined whether this trend differs between conditions by testing if the condition variable (dummy coded as fixed condition $=0$ ) increases model fit compared to the previous best-fitted model. We added random slopes as recommended by Bliese and Ployhart (2002) as long as their addition did not result in a convergence error, or in other words, all models had random intercepts by-participants, and when possible (if convergence), random slopes-by participants across repetitions. We followed the same approach when analyzing the IMTP exercise, with force serving as the dependent variable instead of barvelocity. Conditional $R^{2}$ for mixed regression models was calculated to quantify the explained variance of each model.

Second, we analyzed the mean difference in bar-velocity and mean force of each condition by equalizing the number 
of repetitions. This was done by removing excess repetitions from the analysis. For example, if a participant performed 8 repetitions in the choice condition and 10 in the fixed condition, then the two last repetitions from the fixed condition were removed. We then calculated the mean velocity or force value for each participant in each condition and compared these between the two conditions for each exercise via three separate paired-sample $t$ tests.

\section{Variability in self-selected repetitions}

First, we calculated the mean and SD of the repetitions performed in each exercise. Second, to examine the extent by which participants utilized the provided repetition range (i.e., 8-12), we calculated the percent of each number of repetitions completed per set for each exercise. Finally, to assess the extent by which participants changed the number of repetitions between sets, we calculated whether the number of repetitions was either equal (0) or different (1) between sets for each set in each exercise. We then calculated these proportions for each exercise. We also analyzed whether the number of repetitions differed between the first and second sets in the self-selected condition, by testing a 2 (set: first/second) $\times 3$ (exercise: IMTP/ bench/ squat) repeated-measures analysis of variance (ANOVA).

\section{Self-report measures}

Because the three items of the perceived autonomy scale revealed high reliability (Cronbach's $\alpha=0.72$ and 0.69 in the choice and fixed conditions, respectively) we calculated the mean of the three items for each participant in each condition, and compared the outcome between the two conditions via a paired-sample $t$ test. We compared the enjoyment ratings between the two conditions via a paired-sample $t$ test. We coded participants' responses on the preference scale as "self-selected condition" for positive values, "fixed condition" for negative values, and "no preference" for zero, and calculated the proportion of each. We then compared the proportion of participants preferring either the self-selected or fixed condition, using a binomial test.

Significance was set at $p<0.05$. When testing for the same hypothesis (e.g., mean bar-velocity and force output), $t$ tests were Holm corrected for multiple comparisons. Hedge's $g$ effect size for $t$ tests was calculated based on the mean standard-deviations (Hedge's $g_{\text {av }}$ ). In order to address the possibility that this study was underpowered (among other reasons), we also incorporated Bayesian analyses, which do not require a stopping rule (e.g., Rouder 2014). If a $t$ test yielded a non-significant result, we conducted a Bayesian $t$ test $\left(r_{\text {prior }}=0.707\right)$ (Morey, Rouder, \& Jamil 2015) to assess the extent by which the data was likely under the null hypothesis, compared to a non-zero difference (i.e., a Bayes factor; $\left.\mathrm{BF}_{01}\right)$ (Jarosz \& Wiley 2014). When relevant, 95\% confidence intervals (CIs) are reported. All dependent variables were tested for normality via (1) a visual inspection of the density plots, and (2) kurtosis and skewness inspection, in which skewness $>2$ and kurtosis $>7$ were considered as substantial deviations from normality (West, Finch, \& Curran 1995). Statistical analyses and figures were carried out with $\mathrm{R}$ (version 3.6.1) using the following packages: lme4, lmeTest, piecewiseSEM, moments, BayesFactor, and ggplot2. R syntax for all analyses and data are available at https://bit.ly/2WSeNli.

\section{Results}

The skewness and kurtosis were all smaller than 3 and 7 , respectively, across the performance and psychological values. Therefore, these variables were considered appropriate for the use in parametric tests.

\section{Bar-velocity over repetitions}

In the bench-press, a linear model with a random slope for repetitions yielded the best model-fit (conditional $R^{2}=0.86$ ). The addition of the condition variable increased model-fit ( $p=0.046$, Conditional $R^{2}=0.87$ ), yet no difference was found in the linear slopes between conditions $(b=0.001$, $\mathrm{SE}=0.0006, p=0.085,95 \% \mathrm{CI}[-0.0001,0.002])$. In the squat, a linear model with a random slope for repetitions yielded the best model-fit (conditional $R^{2}=0.90$ ). The addition of the condition variable increased model fit $(p<0.001$, conditional $R^{2}=0.91$ ), yet no difference was found in the linear slopes between conditions $(b=-0.001, \mathrm{SE}=0.0007$, $p=0.146,95 \%$ CI $[-0.002,0.0003]$ ) (see Fig. 1).

\section{Force production over repetitions}

Across conditions, a linear model with a random slope for repetitions yielded the best model-fit $(b=-7.00, \mathrm{SE}=2.73$, $p=0.018,95 \%$ CI $[-12.24,-1.36]$, Conditional $R^{2}=0.99$ ). The addition of the condition variable did not increase model-fit ( $p=0.202$, conditional $R^{2}=0.99$ ).

\section{Variability in self-selected repetitions}

The mean and SD of the number of repetitions completed in the bench press, squat and IMTP exercises were $10.2 \pm 1.6,9.6 \pm 1.4$ and $10.6 \pm 1.6$, respectively. The proportion of selected number of repetitions in each exercise is presented in Fig. 2. Out of the two consecutive sets performed per exercise in the choice condition, in 50\%, $59 \%$ and $50 \%$ of the cases the number of repetitions were the same in the bench-press, squat, and IMTP exercises, 

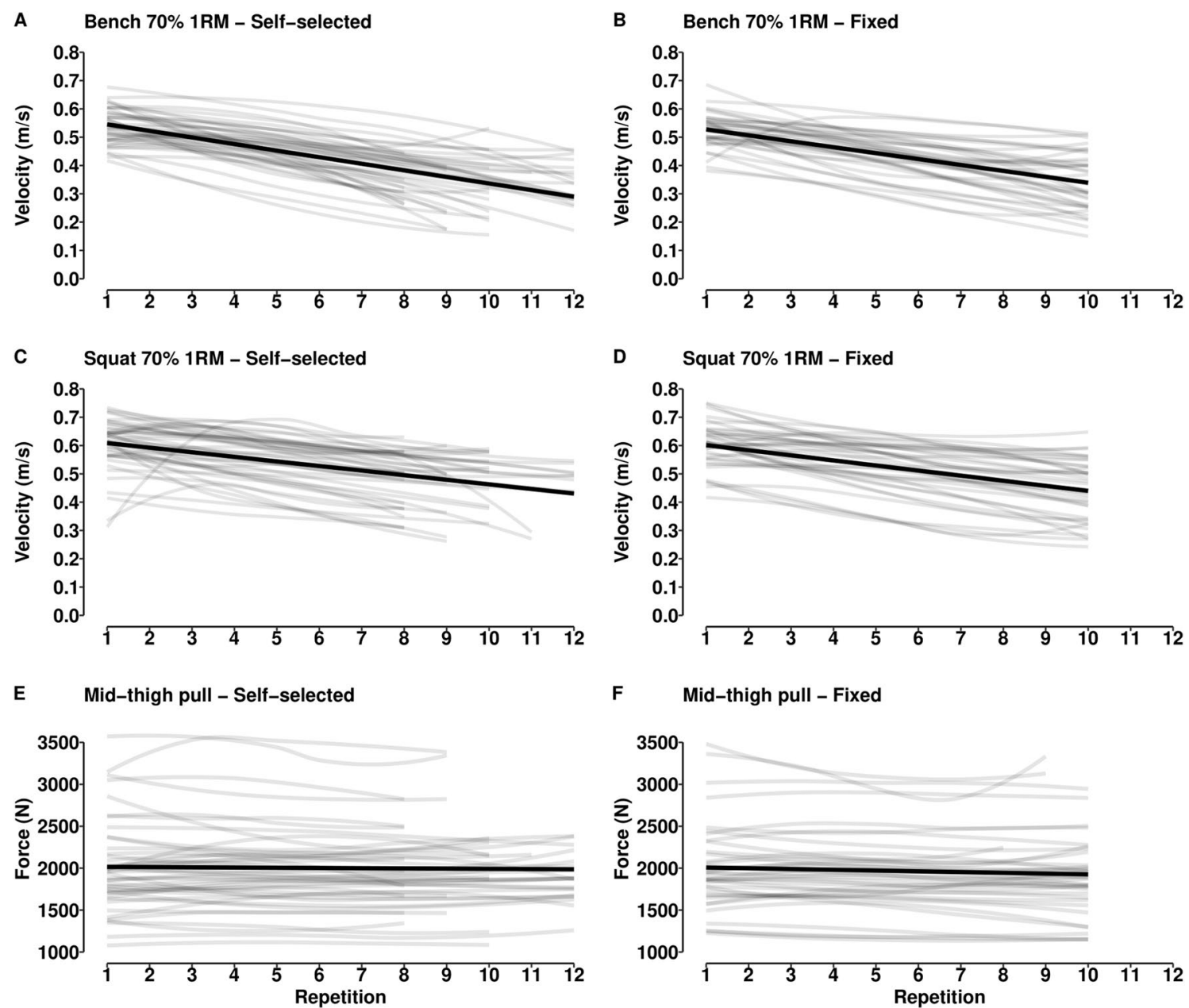

Fig. 1 Distribution of bar-velocity and force over the set in each exercise and condition. The gray lines represent the values of individual participants, fitted using the locally estimated scatterplot smoothing

(LOESS) method, and the fitted regression model in a black line. a-d depict bar-velocity in the bench press and squat in each condition, respectively. e, $\mathbf{f}$ depict force-output in the IMTP in each condition

respectively. The ANOVA comparing the number of repetitions between the first and second sets yielded insignificant effects for either set $(F(1,19)=1.03, p=0.323$, $\left.\eta_{\mathrm{p}}^{2}=0.05\right)$ or the set by exercise interaction $(F(2,38)=0.02$, $\left.p=0.979, \eta_{\mathrm{p}}^{2}=0.001\right)$. This indicated the change in repetitions between the sets did not follow a strong trend for either increasing or decreasing the number of repetitions.

\section{Mean bar-velocity, mean force, enjoyment, perceived autonomy and preferences}

Comparisons between conditions in mean bar-velocity, mean force, enjoyment, perceived autonomy, and preferences are presented in Table 2. Individual ratings of approach preferences are presented in Fig. 3.

\section{Discussion}

To our knowledge, this is the first study to directly compare between two repetitions-prescription approaches: a fixed and predetermined number of repetitions and a selfselected number out of a given range. In both conditions, participants completed two sets of the bench press and squat using $70 \%$ of their 1RM, and two sets of the IMTP. In the fixed condition participants performed 10 repetitions across sets and exercises, whereas in the self-selected condition they selected how many repetitions to perform out of the 8-12 range. Participants reported their perceived autonomy, and enjoyment after each session, and rated their preference for each approach immediately after the last session, and $24 \mathrm{~h}$ afterwards. We found no differences between the two approaches in bar-velocity and force, but observed that participants changed the number of self-selected repetitions in $\sim 50 \%$ of the time between sets, and that participants utilized 

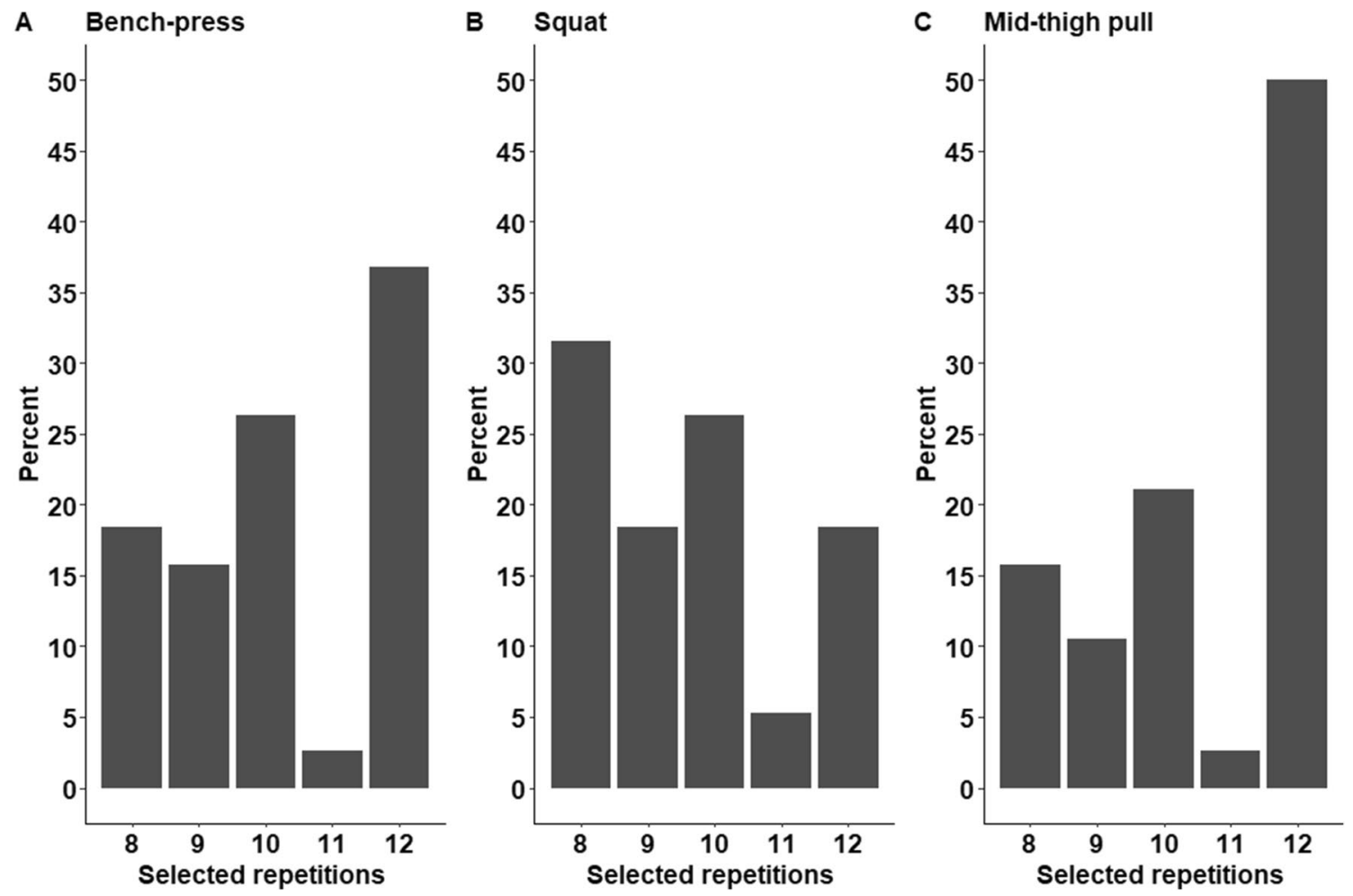

Fig. 2 Distribution of the self-determined repetitions. a-c depict the percent of sets in which each number of repetition was performed from $8-12$, in the bench press, squat and IMTP, respectively

Table 2 Mean \pm SD (minimum and maximum) values of performance and self-report measures of each experimental condition

\begin{tabular}{|c|c|c|c|c|c|c|}
\hline & Fixed condition & Self-selected condition & Mean difference $(95 \% \mathrm{CI})$ & $p$ value & $g^{\prime}$ & $\mathrm{BF}_{01}$ \\
\hline Squat: mean bar-velocity $(\mathrm{m} / \mathrm{s})$ & $0.52 \pm 0.08(0.34,0.62)$ & $0.54 \pm 0.07(0.36,0.63)$ & $-0.02(-0.061,0.008)$ & 0.37 & 0.31 & 2.12 \\
\hline Bench-press: mean bar-velocity $(\mathrm{m} / \mathrm{s})$ & $0.43 \pm 0.06(0.28,0.55)$ & $0.44 \pm 0.06(0.27,0.56)$ & $-0.006(-0.03,0.01)$ & 1.00 & 0.09 & 3.00 \\
\hline IMTP: mean force $(\mathrm{N})$ & $1967 \pm 500(1180,3139)$ & $1969 \pm 538(1154,3470)$ & $-1.77(-66.97,63.43)$ & 1.00 & 0.003 & 3.17 \\
\hline Enjoyment & $77.88 \pm 19.60(35,100)$ & $70.83 \pm 29.86(20,100)$ & $7.05(-10.21,24.32)$ & 0.40 & 0.26 & 2.96 \\
\hline Perceived autonomy & $2.19 \pm 0.733(1,3.75)$ & $3.05 \pm 0.757(1.25,4)$ & $1.05(0.605,1.50)$ & $<0.001$ & 1.48 & - \\
\hline Preference $N(\%)$ & $7(36.84 \%)$ & $10(52.63 \%)$ & - & 0.63 & - & - \\
\hline
\end{tabular}

Mean differences, 95\% confidence intervals (CI), $p$ values, Hedge's $g$ effect sizes, and Bayes factors of the condition-differences are also reported

most of the repetition range. Participants reported similar enjoyment levels in both conditions, and mixed responses concerning the approach they preferred following. However, perceived autonomy was considerably higher in the self-selected condition.

Several reasons might explain the similar performance outcomes observed in both conditions. First, the repetitionsrange in the self-selected condition was at most two repetitions away from the ten repetitions performed in the fixed condition. The similarities between conditions have likely induced similar neuromuscular fatiguing effects leading to the comparable performance outcomes. Second, it is possible that the measurement tools used in the present study lacked the required sensitivity to capture small differences in performance that may exist. While no differences were observed in performance metrics, the number of selected repetitions differed considerably between sets and exercises in the self-selected condition. This indicates that participants took advantage of the option to adjust the number of repetitions within the session, and employed an exercise-regulation strategy (Halperin, Aboodarda, Basset, Byrne, \& Behm 2014a, b). This finding is practically useful as it suggests that letting trainees select the repetitions-number out of a range allows for ongoing intensity-adjustments in RT sessions in view of one's motivation, fatigue, etc. By accounting for this variability, the self-selected approach may optimize RT related adaptations over time. However, this speculation remains to be examined in a longitudinal study. 

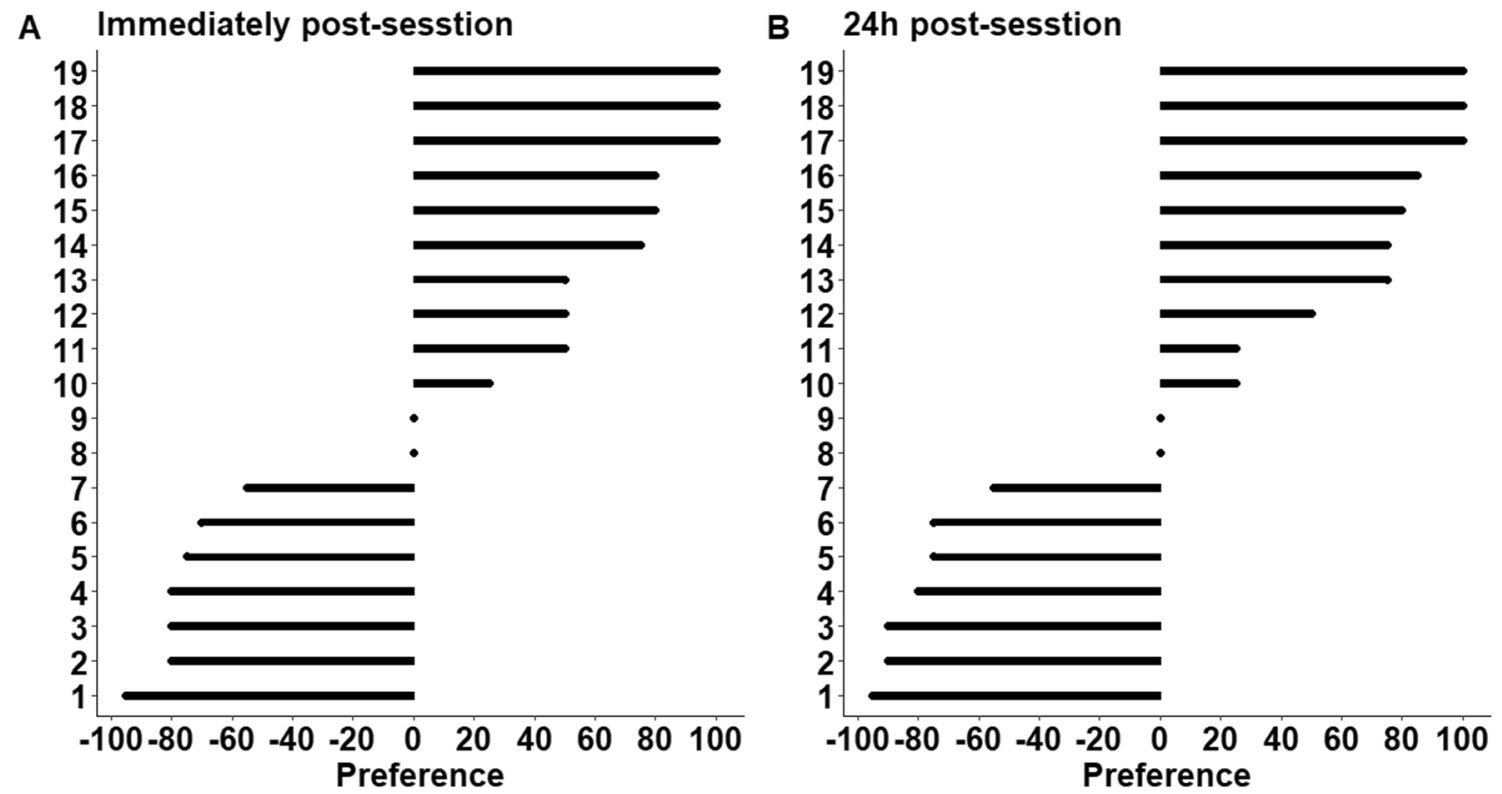

Fig. 3 Extent of preference ratings of each participant for either the fixed approach (negative values; e.g., -100 ) or the self-determined approach (positive values; e.g., 100). Note that participants 8 and 9 rated 0 which indicates they had no preference

From a psychological perspective, the two approaches seemed to have emphasized different motivational components. The fixed approach provided one with a clear taskendpoint but did so at the expense of acting autonomously. Conversely, the self-selected approach provided less clarity about the task-endpoint but allowed participants to act autonomously. Both clear task-endpoints and acting autonomously are factors known to positively influence motivation (Deci and Ryan 2000; Locke \& Latham 1990) and motor performance (Halperin, Aboodarda, Basset, Byrne, \& Behm 2014a; b; Wulf \& Lewthwaite 2016). As such, it may be that some participants were influenced to a greater extent by one of these components. As noted by one participant who preferred the self-determined approach: “...using this approach I can manage my effort with respect to my capacity and goals". Conversely, another participant who preferred the fixed approach noted that: "I find it easier to achieve predetermined objectives than making decisions as I go". This possibility can partly explain the similar enjoyment levels, and the mixed preferences of approaches.

Perception of autonomy was higher in the self-selected condition, indicating that the choice manipulation was effective. Yet, in contrast to other studies (Halperin, Chapman, Martin, Lewthwaite, \& Wulf 2017; Halperin, Wulf, Vigotsky, Schoenfeld, \& Behm 2018; Wulf \& Lewthwaite 2016), we observed no performance-enhancing effects in the self-selected condition. This finding does not necessarily indicate that the autonomy in the self-selected condition did not affect performance, but that the psychological and performance enhancing effects of the fixed approach associated with the clear task-endpoint, may have equalized the effects. We note that despite not observing acute performance and psychological effects, the higher ratings of perceived autonomy can be viewed as a possible advantage of the self-selected approach. This is due to the large body of evidence indicating several positive long-term associations between high autonomy and motor learning (Wulf \& Lewthwaite 2016), exercise adherences (Fortier, Sweet, O'Sullivan, \& Williams 2007; Silva et al. 2011), and positive affect (Deci \& Ryan 2000). The evidence in support of the null hypothesis in most psychological measures suggest both approaches are viable strategies for implementation in the field. However, it remains to be established if similar effects persist over time.

This study has a number of methodological aspects worthy of discussion. The uneven number of repetitions completed in both conditions may have led to some confounding effects. For example, the psychological measures may have been influenced not only by the provided choice, but also by the number of completed repetitions. We attempted to bypass this limitation using a narrow repetition range in the self-selected condition, turning both conditions similar to one another in terms of the number of repetitions completed, and using two different analyses of repetitions. Moreover, the consistent null effects observed in most outcomes suggest that such confounders were less likely to occur. The study consisted of a smaller sample than originally planned, which may have resulted in lower statistical power to detect small to medium sized effects. This did not seem to affect the results, as the findings in almost all measures indicate 
that no-differences were a more likely possibility given the data. We only compared the short-term effects of the two approaches. It is possible that the long-term effects of following one approach or the other will lead to different performance and psychological outcomes. For example, we cannot discount the possibility that some of the observed effects were due to novel aspects associated with the interventions which could wear off or change over time.

In the present study we decided to include three exercises to better represent a training-session, and two sets, to minimize the effects of fatigue. Future studies can also explore if the self-selected condition leads to different outcomes in a more fatiguing session. For example, by reducing the number of exercises (and thereby compounding the effects of neuromuscular fatigue of the same muscle groups) and by including more sets. We also collected the psychological questionnaires only after the sessions were completed. Future studies can also administer the questionnaires before and during the ongoing session, and may include other measures as well, such as the extent of motivation to perform. In addition, future studies should examine the utility of the self-selected approach among other populations as well, especially among untrained individuals. On the one hand, setting a fixed and predetermined number of repetitions that is too difficult to complete can lead to negative affective experiences (e.g., Carver \& Scheier 2002), and subsequently, to fear of failure in future sessions (e.g., Conroy \& Elliot 2004; Englert \& Oudejans 2014). To avoid such situations, the self-selected approach might be viewed as preferable. On the other hand, untrained populations following the selfselected approach may choose to perform the lowest number of repetitions out of a range due to a fear of exerting high effort, or the highest possible number due to a lack of experience. These occurrences may lead to under- or over stimulation of the neuromuscular system.

\section{Conclusion}

To our knowledge, this is the first study to directly compare performance and self-report outcomes between the fixed and self-selected repetitions-prescription approaches, both advocated by leading RT guidelines. The mostly equivalent outcomes in both conditions indicate both approaches are comparable, at least in the short-term. Overall, these findings are practically useful as they suggest that coaches and trainees can follow either one of the approaches based on preferences or certain training goals.

Author contributions IH, AE, and IHN, designed the study, IHN and IIRS collected and coded the data, AE analyzed the data, IH and AE interpreted the data, and wrote the manuscript. All authors have read and approved the final version of the manuscript.

Data and code availability $\mathrm{R}$ syntax and data for all analyses and data are available at https://bit.ly/2WSeNli.

\section{Compliance with ethical standards}

Conflict of interest The authors declare that they have no competing interests.

Ethical approval This study was approved by the Tel-Aviv University Institutional Review Board 2019-0325.

Informed consent The manuscript does not contain clinical studies or patient data. All participants read and signed an informed consent form prior to their participation in this study.

\section{References}

American College of Sports Medicine. (2013). ACSM's guidelines for exercise testing and prescription. USA: Lippincott Williams \& Wilkins.

Billaut, F., Bishop, D. J., Schaerz, S., \& Noakes, T. D. (2011). Influence of knowledge of sprint number on pacing during repeated-sprint exercise. Medicine and Science in Sports and Exercise, 43(4), 665-672.

Bliese, P. D., \& Ployhart, R. E. (2002). Growth modeling using random coefficient models: Model building, testing, and illustrations. Organizational Research Methods, 5(4), 362-387. https://doi. org/10.1177/109442802237116.

Carver, C. S., \& Scheier, M. F. (2002). Control processes and selforganization as complementary principles underlying behavior. Personality and Social Psychology Review, 6(4), 304-315. https ://doi.org/10.1207/S15327957PSPR0604_05.

Comfort, P., Dos'Santos, T., Beckham, G. K., Stone, M. H., Guppy, S. N., \& Haff, G. G. (2019). Standardization and methodological considerations for the isometric midthigh pull. Strength and Conditioning Journal, 41(2), 57-79. https://doi.org/10.1519/ SSC.0000000000000433.

Conroy, D. E., \& Elliot, A. J. (2004). Fear of failure and achievement goals in sport: Addressing the issue of the chicken and the egg. Anxiety, Stress and Coping, 17(3), 271-285.

Deci, E. L., \& Ryan, R. M. (2000). The "what" and "why" of goal pursuits: Human needs and the self-determination of behavior. Psychological Inquiry, 11(4), 227-268.

DeLorme, T. L. (1945). Restoration of muscle power by heavy-resistance exercises. JBJS, 27(4), 645-667.

Englert, C., \& Oudejans, R. R. (2014). Is choking under pressure a consequence of skill-focus or increased distractibility? Results from a tennis serve task. Psychology, 5(9), 1035-1043.

Fortier, M. S., Sweet, S. N., O’Sullivan, T. L., \& Williams, G. C. (2007). A self-determination process model of physical activity adoption in the context of a randomized controlled trial. Psychology of Sport and Exercise, 8(5), 741-757.

Fragala, M. S., Cadore, E. L., Dorgo, S., Izquierdo, M., Kraemer, W. J., Peterson, M. D., et al. (2019). Resistance training for older adults: position statement from the National strength and conditioning association. The Journal of Strength and Conditioning Research, 33(8), 2019-2052.

Halperin, I., Aboodarda, S. J., Basset, F. A., Byrne, J. M., \& Behm, D. G. (2014a). Pacing strategies during repeated maximal voluntary 
contractions. European Journal of Applied Physiology, 114(7), 1413-1420. https://doi.org/10.1007/s00421-014-2872-3.

Halperin, I., Aboodarda, S. J., Basset, F. A., \& Behm, D. G. (2014b). Knowledge of repetitions range affects force production in trained females. Journal of Sports Science and Medicine, 13(4), 736.

Halperin, I., Chapman, D. W., Martin, D. T., Lewthwaite, R., \& Wulf, G. (2017). Choices enhance punching performance of competitive kickboxers. Psychological Research Psychologische Forschung, 81(5), 1051-1058.

Halperin, I., Wulf, G., Vigotsky, A. D., Schoenfeld, B. J., \& Behm, D. G. (2018). Autonomy: A missing ingredient of a successful program? Strength and Conditioning Journal, 40(4), 18-25.

Hanson, N. J., \& Buckworth, J. (2015). The effect of endpoint knowledge on perceived exertion, affect, and attentional focus during self-paced running. The Journal of Strength and Conditioning Research, 29(4), 934-941.

Jarosz, A. F., \& Wiley, J. (2014). What are the odds? A practical guide to computing and reporting Bayes factors. The Journal of Problem Solving, 7(1), 2.

Locke, E. A., \& Latham, G. P. (1990). A theory of goal setting \& task performance. USA: Prentice-Hall Inc.

Morey, R. D., Rouder, J. N., \& Jamil, T. (2015). BayesFactor: Computation of Bayes factors for common designs. R Package Version $0.9,9,2014$

Nelson, M. E., Rejeski, W. J., Blair, S. N., Duncan, P. W., Judge, J. O., King, A. C., et al. (2007). Physical activity and public health in older adults: recommendation from the American College of Sports Medicine and the American Heart Association. Medicine and Science in Sports and Exercise, 39(8), 1435-1445.

Richens, B., \& Cleather, D. J. (2014). The relationship between the number of repetitions performed at given intensities is different in endurance and strength trained athletes. Biology of Sport, 31(2), 157.

Rouder, J. N. (2014). Optional stopping: No problem for Bayesians. Psychonomic Bulletin and Review, 21(2), 301-308.

Ryan, R. M., \& Deci, E. L. (2002). An overview of self-determination theory: An organismic-dialectical perspective. In E. L. Deci \& R. M. Ryan (Eds.), Handbook of self-determination research (pp. 3-33). Rochester: The University of Rochester Press.

Ryan, R. M., Rigby, C. S., \& Przybylski, A. (2006). The motivational pull of video games: A self-determination theory approach. Motivation and Emotion, 30(4), 344-360.

Sands, W. A., Wurth, J. J., \& Hewit, J. K. (2012). The national strength and conditioning association's (NSCA) basics of strength and conditioning manual. NSCA, Editor: NSCA.

Sanli, E. A., Patterson, J. T., Bray, S. R., \& Lee, T. D. (2013). Understanding self-controlled motor learning protocols through the selfdetermination theory. Frontiers in Psychology, 3, 611.

Shimano, T., Kraemer, W. J., Spiering, B. A., Volek, J. S., Hatfield, D. L., Silvestre, R., et al. (2006). Relationship between the number of repetitions and selected percentages of one repetition maximum in free weight exercises in trained and untrained men. The Journal of Strength and Conditioning Research, 20(4), 819-823.

Silva, M. N., Markland, D., Carraca, E. V., Vieira, P. N., Coutinho, S. R., Minderico, C. S., et al. (2011). Exercise autonomous motivation predicts 3-yr weight loss in women. Medicine and Science in Sports and Exercise, 43(4), 728-737.

Smith, K. G., Locke, E. A., \& Barry, D. (1990). Goal setting, planning, and organizational performance: An experimental simulation. Organizational Behavior and Human Decision Processes, 46(1), 118-134.

Standage, M., Duda, J. L., \& Ntoumanis, N. (2005). A test of selfdetermination theory in school physical education. British Journal of Educational Psychology, 75(3), 411-433.

Stanley, D. M., Williams, S. E., \& Cumming, J. (2009). Preliminary validation of a single-item measure of exercise enjoyment: The Exercise Enjoyment Scale. Journal of Sport \& Exercise Psychology, 31, S138-S139.

Teixeira, P. J., Carraça, E. V., Markland, D., Silva, M. N., \& Ryan, R. M. (2012). Exercise, physical activity, and self-determination theory: a systematic review. International Journal of Behavioral Nutrition and Physical Activity, 9(1), 78.

Todd, J. S., Shurley, J. P., \& Todd, T. C. (2012). Thomas L. DeLorme and the science of progressive resistance exercise. Journal of Strength and Conditioning Research, 26(11), 2913-2923. https ://doi.org/10.1519/JSC.0b013e31825adcb4.

Vlachopoulos, S. P., Ntoumanis, N., \& Smith, A. L. (2010). The basic psychological needs in exercise scale: Translation and evidence for cross-cultural validity. International Journal of Sport and Exercise Psychology, 8(4), 394-412. https://doi.org/10.1080/16121 97X.2010.9671960.

West, S. G., Finch, J. F., \& Curran, P. J. (1995). Structural equation models with non-normal variables: Problems and remedies. In R. H. Hoyle (Ed.), Structural equation modelling: Concepts, issues and applications (pp. 56-75). Thousand Oaks: Sage.

Wingfield, G., Marino, F., \& Skein, M. (2018). The influence of knowledge of performance endpoint on pacing strategies, perception of effort, and neural activity during $30-\mathrm{km}$ cycling time trials. Physiological Reports, 6(21), e13892.

Wulf, G., \& Lewthwaite, R. (2016). Optimizing performance through intrinsic motivation and attention for learning: The OPTIMAL theory of motor learning. Psychonomic Bulletin and Review, 23(5), 1382-1414.

Publisher's Note Springer Nature remains neutral with regard to jurisdictional claims in published maps and institutional affiliations. 